\title{
Structure and Magnetic Properties of Gd/Ti Nanoscale Multilayers
}

\author{
A.V. Svalov ${ }^{1,2, a}$, V.O. Vas'kovskiy ${ }^{1}$, J.M. Barandiarán ${ }^{2}$, K.G. Balymov", \\ A.N. Sorokin ${ }^{1}$, I. Orue ${ }^{3}$, A. Larrañaga ${ }^{3}$, N.N. Schegoleva ${ }^{4}$ and \\ G.V. Kurlyandskaya ${ }^{2, b}$ \\ ${ }^{1}$ Ural State University, 620083, Ekaterinburg, Russia \\ ${ }^{2}$ Departamento de Electricidad y Electrónica, Universidad del País Vasco, 48080, Bilbao, Spain \\ ${ }^{3}$ SGlker, Servicios Generales de Investigación, Universidad del País Vasco, 48080, Bilbao, Spain \\ ${ }^{4}$ Institute of Metal Physics, Ural Branch of RAS, 620990, Ekaterinburg, Russia \\ a andrey.svalov@usu.ru, bgalina@we.lc.ehu.es
}

Keywords: Gadolinium, multilayers, magnetization, magnetic ordering temperature.

\begin{abstract}
Structure and magnetic properties of nanoscale $[\mathrm{Gd} / \mathrm{Ti}]_{\mathrm{n}}$ multilayers prepared by rfsputtering are studied. It is found that the decrease in the $\mathrm{Gd}$ layer thickness $L_{\mathrm{Gd}}$ leads to beginning of the structure transformation in Gd layers from the fine-crystalline to amorphous state when $L_{\mathrm{Gd}}$ becomes less than $2 \mathrm{~nm}$. The Curie temperature $T_{\mathrm{C}}$ decreases as a function of the Gd layer thickness in the same way as in early studied epitaxially grown Gd films, i.e. in case for which the finite-size effect plays most important role. A deviation of the $T_{\mathrm{C}}\left(L_{\mathrm{Gd}}\right)$ behaviour at very low $L_{\mathrm{Gd}}$ from the fit according to the finite-size law is probably caused by the island-like structure of the Gd layers.
\end{abstract}

\section{Introduction}

The rare earth metals have for a long time been of great scientific and technological interest due to their unique magnetic properties [1,2]. Recently, gadolinium thin films began to be in use for spintronics devices [3] or magnetic refrigeration [4]. However magnetic properties of materials in thin film state may differ from properties of the bulk materials $[5,6]$. It has been found that, as the film thickness goes down, the Curie temperature $T_{\mathrm{C}}$ of thin ferromagnetic films decreases [7]. In particular, it was observed that the Gd films epitaxially grown on the inert substrates show a remarkable depression of $T_{\mathrm{C}}$ following the finite-size scaling [8]. Special sensitivity of the Gd film properties to deposition parameters was also reported [8]. Therefore, the structure and properties of the Gd films prepared by the rf-sputtering method, which is well adapted to technological applications, can be different in comparison with the epitaxially grown films.

In this work, both structural features and magnetic properties were studied on the $\mathrm{Gd} / \mathrm{Ti}$ sputtered nanoscale multilayers with different thickness of Gd layers.

\section{Experimental}

The $\mathrm{Gd} / \mathrm{Ti}$ multilayers were prepared by alternative rf-sputtering deposition of Gd layers and $\mathrm{Ti}$ spacers onto glass substrates. The base pressure in the chamber was $3 \times 10^{-7}$ mbar and argon gas flow with a pressure of $5 \times 10^{-3} \mathrm{mbar}$ was used for the sputtering process. The deposition rate was about $0.15 \mathrm{~nm} / \mathrm{s}$ for $\mathrm{Gd}$ and about $0.07 \mathrm{~nm} / \mathrm{s}$ for Ti. The thicknesses of the Gd layers $\left(L_{\mathrm{Gd}}\right)$ were varied from $0.9 \mathrm{~nm}$ to $12 \mathrm{~nm}$ and the thickness of Ti non-magnetic spacers was kept constant $(2 \mathrm{~nm})$. Ti was chosen for the spacers as a material with low solubility in rare earth materials. Each sample had a protective buffer and coating layers of nonmagnetic materials.

The structural state of the samples was examined by transmission electron microscopy (TEM) and X-ray diffraction (XRD). For TEM investigations the samples with a thickness of $50 \mathrm{~nm}$ were deposited onto the cleavage surface of $\mathrm{NaCl}$ crystals. Low angle XRD was used to determine the 


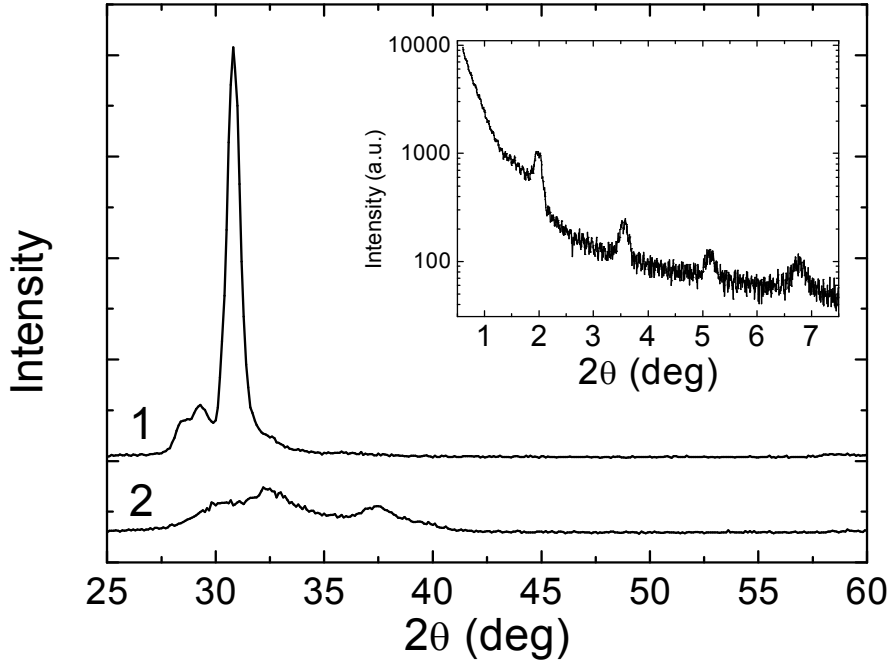

Fig. 1. X-ray diffractograms for $[\mathrm{Gd}(12 \mathrm{~nm}) / \mathrm{Ti}]_{10}(1)$ and $[\mathrm{Gd}(1.5 \mathrm{~nm}) / \mathrm{Ti}]_{50}(2)$ multilayers. Inset: low angle $\mathrm{X}$-ray diffraction for $[\mathrm{Gd}(3 \mathrm{~nm}) / \mathrm{Ti}]_{30}$ sample.
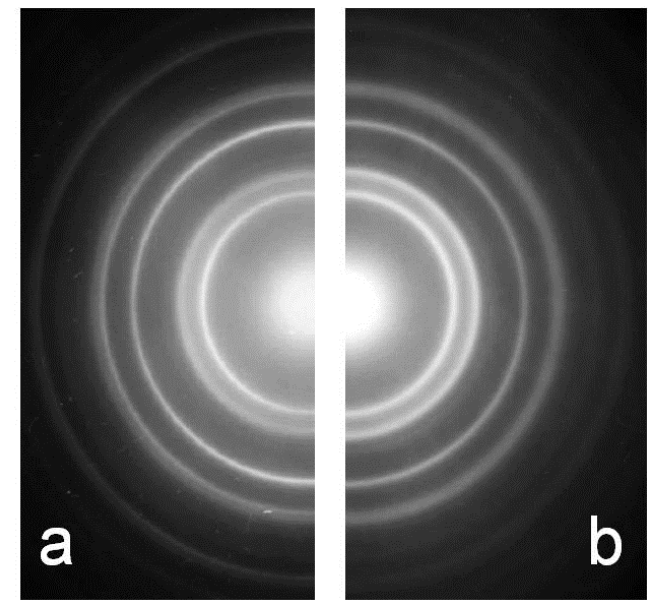

Fig. 2. Selected area of TEM diffraction patterns for $[\mathrm{Gd}(3 \mathrm{~nm}) / \mathrm{Ti}]_{10} \quad$ (a) and $[\mathrm{Gd}(1.5 \mathrm{~nm}) / \mathrm{Ti}]_{14}$ (b) multilayers.

quality of the layers. Magnetic measurements were performed using a SQUID magnetometer and a Quantum design physical property measurement system.

\section{Results and discussion}

Structure. The low angle XRD patterns for all multilayers showed Bragg peaks associated with the periodic structure of the multilayers. Inset in Fig. 1 shows an example of the low-angle X ray diffractogram for the $\mathrm{Gd}(3 \mathrm{~nm}) / \mathrm{Ti}]_{30}$ sample. The observed peaks allow the determination of the layer thickness which agrees well with that expected from the deposition time. The presence of the Bragg peaks in the diffractograms of the samples even with the smallest Gd layers of $0.9 \mathrm{~nm}$ thickness demonstrates the existence of a well defined layered structure in all cases under consideration.

According to the XRD and TEM data, the structure of Gd layers in the studied samples is a hexagonal close-packed one. Fig. 1 shows the selected $X$ ray spectra for the Gd/Ti samples with different thicknesses of Gd layers. The nanocrystalline multilayers have a strong texture: only the Gd hep (002) peak is clearly visible in the XRD patterns (Fig.1, curve 1). The decrease of the Gd layer thickness is accompanied by the increase of the peak widths. For all samples with $L_{\mathrm{Gd}}>1.5$ $\mathrm{nm}$, the grain sizes, $d$, were calculated using the familiar Scherer's equation:

$$
d=0.9 \lambda / B \cos \theta_{\mathrm{B}}
$$

where $\lambda$ is the radiation wavelength, $B$ is the full width of the $\mathrm{X}$ ray peak at half maximum and $\theta_{\mathrm{B}}$ is the position of the peak. For these samples, the Gd grain size was estimated as equal to the thickness of the Gd layers. For multilayers with $L_{\mathrm{Gd}}$ of $1.5 \mathrm{~nm}$ and $0.9 \mathrm{~nm}$, the diffraction peak transforms into an amorphous like halo (Fig. 1, curve 2). In this case, it is not possible to use the Scherer's equation. For $L_{\mathrm{Gd}} \sim 3 \mathrm{~nm}$ and below, it was difficult to distinguish between the very finegrained nanocrystaline or amorphous state of the Gd layers. This conclusion is supported by the TEM data. On the one hand, the well pronounced diffraction rings are visible, on the other hand, their width evidences that the correlation length is small (Fig. 2). Moreover, a tendency to increasing the width of diffraction rings is observed upon decrease of $L_{\mathrm{Gd}}$. 


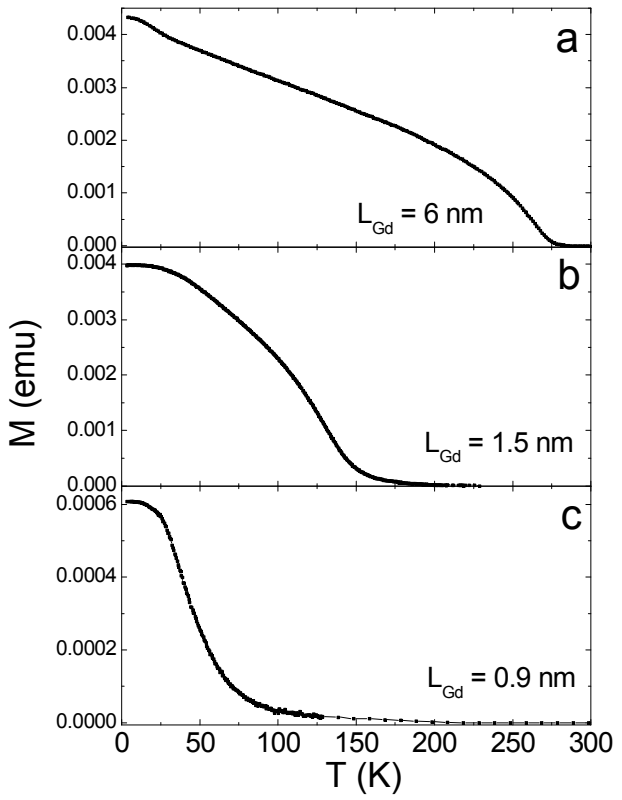

Fig. 3. Temperature dependence of magnetization for selected $[\mathrm{Gd} / \mathrm{Ti}]_{\mathrm{n}}$ multilayers with different $L_{\mathrm{Gd}}$ value.

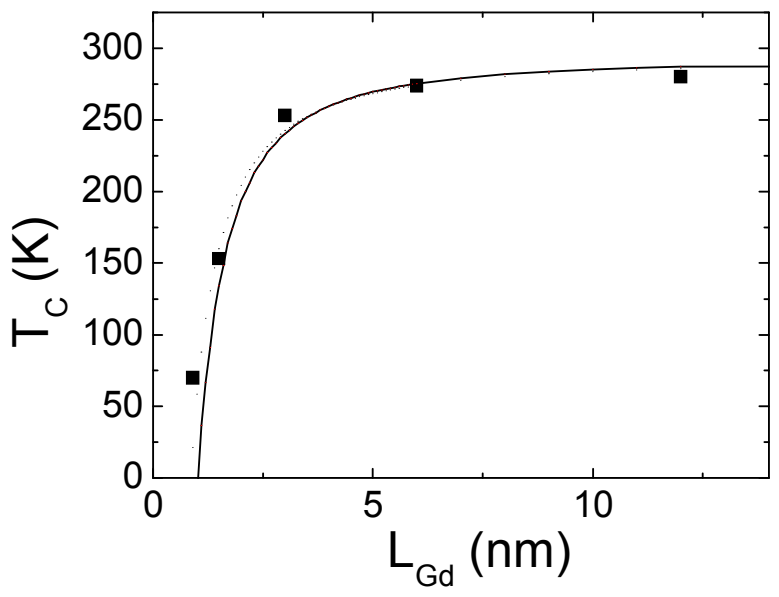

Fig. 4. Dependence of magnetic ordering temperature $T_{\mathrm{C}}$ on the Gd layer thickness for $[\mathrm{Gd} / \mathrm{Ti}]_{\mathrm{n}}$ multilayers. Squares: experimental data. Line: fit according to Eq. (2) using the fitting parameters from [8].

Magnetic properties. Fig. 3 shows the temperature dependences of magnetization $M$ for the selected $[\mathrm{Gd} / \mathrm{Ti}]_{\mathrm{n}}$ multilayers with different $L_{\mathrm{Gd}}$ value. The measuring field was 120 Oe. The temperature $T_{\mathrm{C}}$ was estimated from the $M(T)$ curves by linear extrapolation of the high-temperature parts to the zero value of $M$. Fig. 4 shows a summary of $T_{\mathrm{C}}$ as a function of magnetic layer thickness. For $L_{\mathrm{Gd}}$ below $5 \mathrm{~nm}$ the $T_{\mathrm{C}}$ decreases sharply. A similar dependence on the film thickness was found earlier for high-quality epitaxially grown Gd films [8].

The decrease of the magnetic ordering temperature for the multilayers studied in the present work can be caused by both the size effect and distortions of crystalline lattice [9]. It may happen due to inhomogeneous strains and conversion of the fine-crystalline structure into the amorphous state with the Gd layer thickness decrease. According to the finite-size scaling theory [10], the shift of the Curie temperature in magnetic films can be described approximately by the power law

$$
\left[T_{\mathrm{C}}(\infty)-T_{\mathrm{C}}(L)\right] / T_{\mathrm{C}}(\infty)=C \cdot L^{-\lambda}
$$

where $T_{\mathrm{C}}(\infty)$ is the Curie temperature for bulk material, $T_{\mathrm{C}}(L)$ is the Curie temperature for thin film with a thickness $L, C$ is the constant, and $\lambda$ is the shift exponent. The line (Fig. 4) shows a fit of the experimental $T_{\mathrm{C}}$ for the Gd/Ti multilayers using Eq. (2) with the $C$ and $\lambda$ values taken from Ref. 8 , where Gd films were prepared by epitaxial layer-by-layer deposition with the consequent proper annealing cycle for the accommodation of misfit dislocations. It is seen, that the experimental $T_{\mathrm{C}}\left(L_{\mathrm{Gd}}\right)$ dependence for the sputtered films is well described by Eq. (2) using the fitting parameters for the low defective epitaxially grown films with the thicknesses down to $\sim 1.5 \mathrm{~nm}$. However, at $L_{\mathrm{Gd}}=0.9 \mathrm{~nm}$ the experimental $T_{\mathrm{C}}$ value significantly exceeds the $T_{\mathrm{C}}$ extrapolated from the curve following Eq (2). This means that at $L_{\mathrm{Gd}}=0.9 \mathrm{~nm}$, the Gd layers do not grow as continuous film but rather like the islands. The real thickness of these islands must be of about $1.2 \mathrm{~nm}$, according to finite-size scaling curve.

The supposition on the island-like structure of the Gd layers finds confirmation from the $M(H)$ curves. Fig. 5 shows the $M(H)$ curves measured in the plane of the samples at $T=2 \mathrm{~K}$. All curves are similar except the one for $L_{\mathrm{Gd}}=0.9 \mathrm{~nm}$. The shape of the later curve can be traceable to an increase of the magnetization axis dispersion caused by the island formation. 

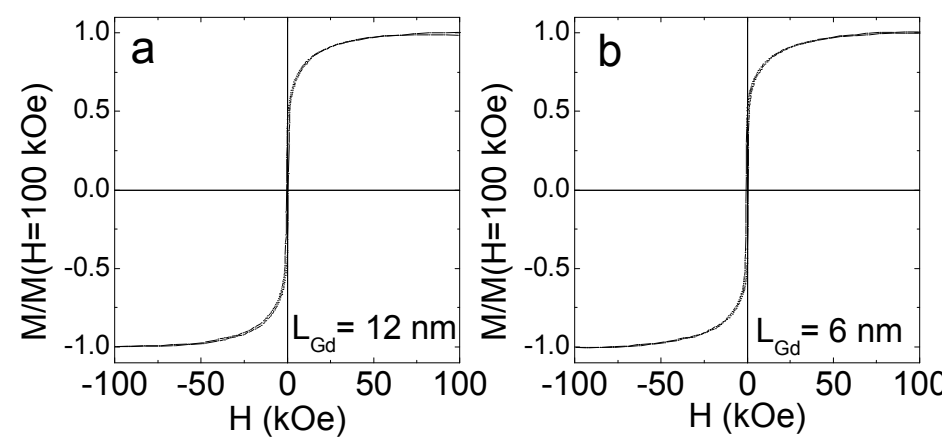

\section{Summary}

The obtained results demonstrate that the finite-size scaling law is valid for thin nanocrystalline films prepared by the rfsputtering method with a thickness of the Gd components above $1.5 \mathrm{~nm}$. The polycrystallinity and the presence of imperfections in the structure do not make an additional contribution to such
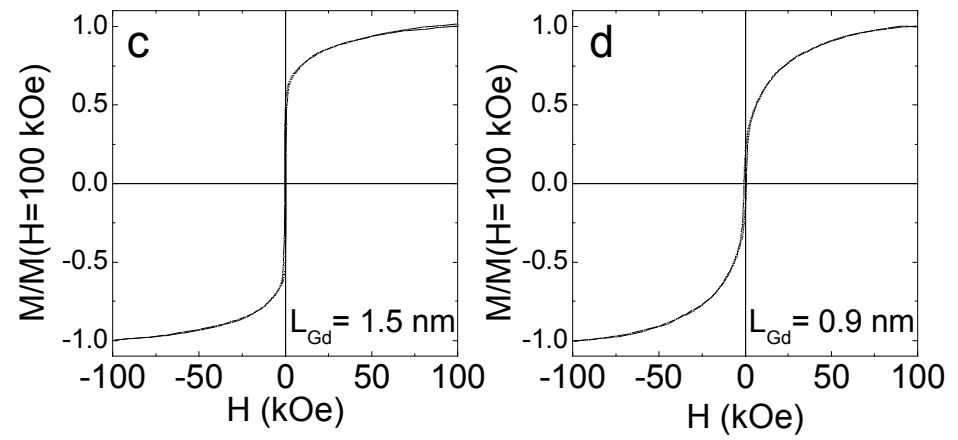

an important parameter of the ferromagnets as the $T_{\mathrm{C}}$ value. The deviation of the $T_{\mathrm{C}}\left(L_{\mathrm{Gd}}\right)$ behavior from the fit according to thefinite-size scaling at very small thicknesses is caused most likely by the formation of the island structure in the course of preparation of the $[\mathrm{Gd} / \mathrm{Ti}]_{\mathrm{n}}$ multilayers rather than the deposition of continuous Gd layers.

Fig. 5. $M(H)$ curves for the selected $[\mathrm{Gd} / \mathrm{Ti}]_{\mathrm{n}}$ multilayers with different $L_{\mathrm{Gd}}$ value measured at $T=2 \mathrm{~K}$.

\section{Acknowledgment}

This work was supported by RFBR (grant 08-02-99063-r_ofi) and Spanish MEC (project MAT2008-06542-C04_02). Technical and human support provided by SGIker X-ray diffraction and magnetic measurements services of UPVEHU is gratefully acknowledged.

\section{References}

[1] S.A. Nikitin: Magnetic Properties of Rare-Earth Metals and Alloys (MSU, Moscow 1989) [in Russian].

[2] J. Jensen and A.R. Mackintosh: Rare Earth Magnetism: Structures and Excitations (Clarendon press, Oxford 1991).

[3] Ya.-H. Fan and H. Brückl: Appl. Phys. Lett. Vol. 83 (2003) p. 3138.

[4] C.W. Miller, D.V. Williams, N.S. Bingham, H. Srikanth: J. Appl. Phys. Vol. 107 (2010) p. 09A903.

[5] C.A.F. Vaz, J.A.C. Bland, G. Lauhoff: Rep. Prog. Phys. Vol. 71 (2008) p. 056501.

[6] A.V. Svalov, V.O. Vas'kovskiy, G.V. Kurlyandskaya, J.M. Barandiarán, N.N. Schegoleva, A.N. Sorokin: Chin. Phys. Lett. Vol. 23 (2006) p. 196.

[7] X.Y. Lang, W.T. Zheng, Q. Jiang: Phys. Rev. B Vol. 73 (2006) p. 224444.

[8] M. Farle, K. Baberschke, U. Stetter, A. Aspelmeier, F. Gerhardter: Phys. Rev. B Vol. 47 (1993) p. 11571.

[9] I. Turek, J. Kudrnovský, G. Bihlmayer, S. Blügel: J. Phys.: Condens. Matter Vol. 15 (2003) p. 2771.

[10] G.A.T. Allan: Phys. Rev. B Vol. 1 (1970) p. 352. 